La radioastronomie à basse

fréquence vit aujourd'hui

sa renaissance à travers la

construction du Square Kilometre

Array (SKA). Deux réseaux

de milliers d'antennes, opérant

dans les domaines de longueur

d'onde métriques et centimétriques,

permettront de cartographier

les différentes phases de l'histoire

de l'Univers, de l'apparition

des premières sources lumineuses

jusqu'aux systèmes d'astres

évolués que nous observons

aujourd'hui.

Mais SKA n'est pas seulement

un grand observatoire

astronomique : il s'agit d'un projet

international qui fait face à des

défis considérables, en bénéficiant

de la plus grande variété

de cultures et de compétences

du monde entier.

\title{
Le Square Kilometre Array (SKA) : un radiotélescope géant pour étudier l'aube et l'évolution du cosmos
}

Chiara Ferrari (chiara.ferrari@oca.eu)

Astronome à l'Observatoire de la Côte d'Azur - Directrice de la Maison SKA-France

OCA, Boulevard de l'Observatoire, 06304 Nice Cedex 4

\section{Un projet global}

Le Square Kilometre Array (SKA), réseau interférométrique qui observera le ciel radio dans les bandes métriques à centimétriques, est l'un des projets majeurs de l'astronomie au sol au niveau mondial. Sa conception originale remonte au tout début des années 1990. C'est à cette époque qu'est née l'idée de construire un télescope capable de mesurer l'évolution du contenu en gaz d'hydrogène atomique neutre (HI) des galaxies au cours de l'histoire de l'Univers. L'observatoire astronomique en question devait forcément être un radiotélescope, les grands nuages d'HI produisant un rayonnement électromagnétique à la longueur d'onde de $21 \mathrm{~cm}$. Depuis son début, le concept de SKA repose sur un réseau d'antennes de taille très importante (de surface collectrice totale d'un kilomètre carré, d'où son nom), car sa capacité à détecter des objets extrêmement faibles et à les séparer spatialement entre eux dépend respectivement de la surface collectrice totale des antennes et de leur distance maximale.

SKA est un projet global nécessitant des efforts conjoints au niveau mondial. Aujourd'hui,il s'agit d'un projet planétaire ${ }^{(\mathrm{a})}$, dont la construction sera lancée en 2021 et qui sera piloté par une organisation intergouvernementale. Au-delà de l'étude du gaz d'HI dans les galaxies, SKA ouvrira l'observation radio sur de vastes domaines de l'astrophysique, de la cosmologie et de la physique fondamentale : l'aube du cosmos, les sources radio transitoires, les objets compacts, les champs magnétiques, les études planétaires, la détection, le suivi électromagnétique d'ondes gravitationnelles... et, surtout, la recherche de l'inconnu. Sur certaines thématiques, cet instrument sera le seul capable de progrès décisifs ; il apportera sur d'autres des informations complémentaires indispensables à de grands observatoires modernes, couvrant des domaines de fréquence différents du spectre électromagnétique.

\section{Deux télescopes pour un observatoire}

Depuis le début, SKA a été conçu pour ouvrir un espace de découverte unique au travers de la combinaison de caractéristiques (sensibilité, champ de vue, résolution, couverture en fréquence) qui seront, dans la plupart des cas, d'un ordre de grandeur supérieur à celles des instruments radio à basse et moyenne fréquence existants ${ }^{(b)}$. Une capacité sans égale d'obtenir des images très nettes avec des temps de pose assez courts (fig. 1) permettra à ce nouvel observatoire de balayer le ciel rapidement et en profondeur. Les grands projets d'observation du ciel qui sont en train d'être conçus avec SKA sont sensés former une " pyramide ", allant d'un relevé moins profond de tout le ciel observable depuis l'hémisphère Sud (environ 31000 degrés carrés) à des régions observées de surface de plus en plus réduite, mais avec une sensibilité croissante. Même pour le relevé le moins profond et le plus étendu, SKA va atteindre les mêmes limites de sensibilité 

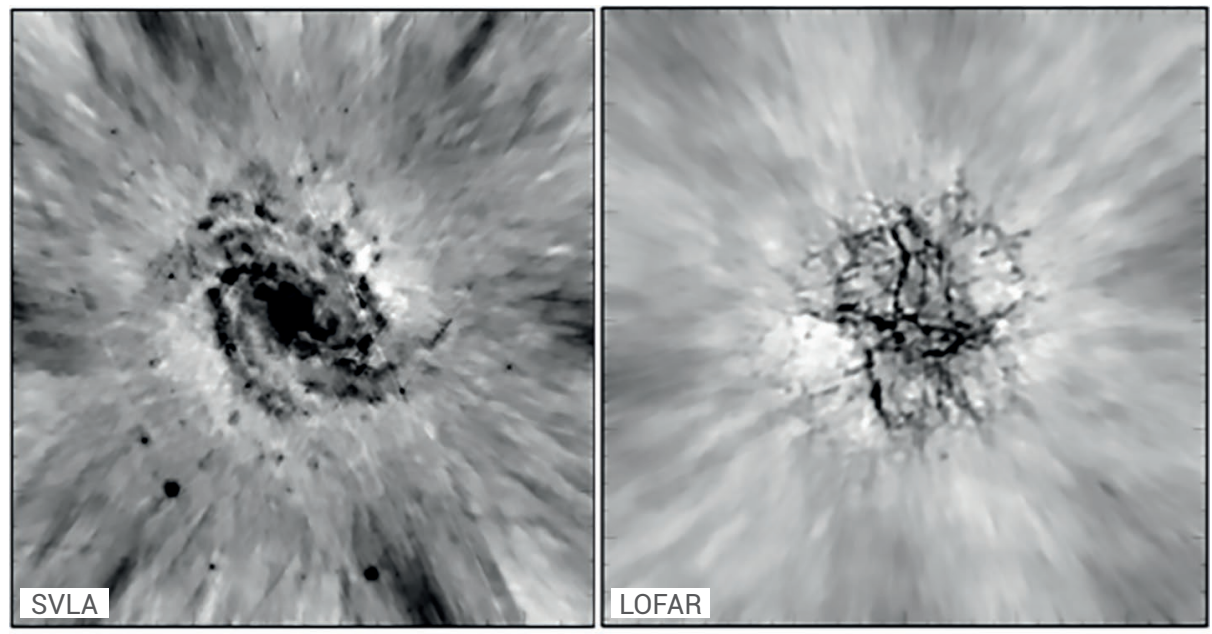

que les instruments actuels obtiennent sur des champs de vue bien inférieurs au degré carré !

Pour atteindre ces performances, l'observatoire SKA sera initialement constitué par deux réseaux d'antennes assez différents en fonction de la bande de longueur d'onde observée (fig. 2). Un peu moins de 200 paraboles de $15 \mathrm{~m}$, avec une couverture en fréquence entre $350 \mathrm{MHz}$ et $15,5 \mathrm{GHz}$ (SKA1-MID), seront installées dans les déserts du Karoo (Afrique du Sud). En revanche, les fréquences entre 50 et $350 \mathrm{MHz}$ (SKA1-LOW) seront observées par un peu plus de 131000 antennes logpériodiques localisées dans le désert de Murchinson (Australie). Ces antennes, similaires aux antennes de télévision en étant constituées de dipôles demi-longueur d'onde de différentes dimensions espacés le long d'une structure de support, seront regroupées en 512 stations. SKA1-LOW se base sur le concept de "réseau d'ouverture", car les signaux des 256 antennes à l'intérieur de chaque station seront d'abord déphasés et additionnés entre eux pour former un ou plusieurs faisceaux par station, ce qui justifie l'appellation "Software Telescopes" pour ce type d'instrument. La distance maximale entre les antennes sera de $150 \mathrm{~km}$ pour SKA1-MID et de $65 \mathrm{~km}$ pour SKA1-LOW.

Après un certain nombre de prétraitements, les énormes taux de données (des milliers de milliards d'octets par seconde) en provenance de toutes les paraboles de SKA1-MID, ou stations dans le cas de
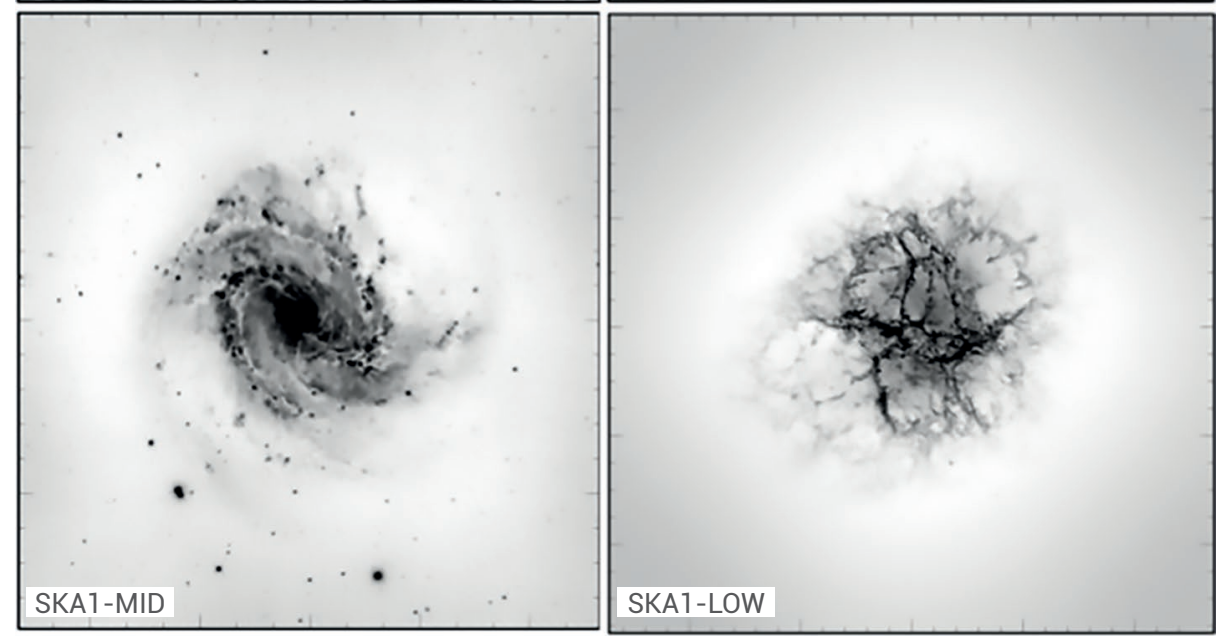

1. Simulations d'observations d'objets complexes : la galaxie spirale M83 dans la colonne de gauche et la nébuleuse du Crabe - reste de supernova résultant de l'explosion d'une étoile massive - à droite. Les performances en termes d'images brutes attendues par deux télescopes qui sont l'état de l'art aujourd'hui sont illustrées en haut (JVLA à gauche, LOFAR à droite). Dans le cas de SKA1-MID (gauche) et de SKA1-LOW (droite) elles sont montrées en bas. II s'agit de simulations d'observations très rapides (de l'ordre de quelques minutes) de ces objets célestes dans une bande de fréquence de $40 \mathrm{MHz}$ centrée autour de 1,4 GHz (gauche) et $140 \mathrm{MHz}$ (droite). D'après R. Braun et al., « Anticipated SKA1 Science Performance », SKA-TEL-SKO-0000818 (2017).

SKA1-LOW, seront combinés soit pour créer de grandes images multifréquences du ciel (au travers de la combinaison interférométrique des $\left.\operatorname{signaux}^{(\mathrm{c})}\right)$, soit pour enregistrer les variations d'intensité en fonction du temps et de la fréquence des signaux radio en provenance de centaines de régions du ciel simultanément (addition phasée des signaux). Il faut souligner que le traitement des données de SKA nécessitera des puissances de calcul de l'ordre de centaines de pétaFLOPS (i.e. environ $10^{17}$ opérations par seconde).

Au-delà des détails techniques concernant les observations radio [1], il est intéressant de comprendre pourquoi des capteurs si différents seront utilisés dans le cas de SKA. Aux plus basses fréquences, un des grands avantages d'utiliser des réseaux est que leur ouverture efficace est proportionnelle à $N_{A} \lambda^{2}$ (où $N_{A}$ est le nombre total d'antennes et $\lambda$ la longueur d'onde d'observation). Avec le nombre important d'antennes simples que nous pouvons installer à un cout raisonnable, l'utilisation de cette technologie aux longueurs d'ondes métriques assure donc une ouverture efficace très élevée et, par conséquent, une grande sensibilité. Dans ce régime de fréquence, un ensemble d'antennes paraboliques, chacune avec une ouverture efficace proportionnelle à sa surface géométrique, ne pourrait jamais entrer en compétition. La réciproque est applicable à des fréquences plus élevées : à partir de plusieurs centaines de $\mathrm{MHz}$, cela nécessiterait des réseaux d'ouverture avec une densité d'antennes tellement élevée que cette solution aurait des couts de 


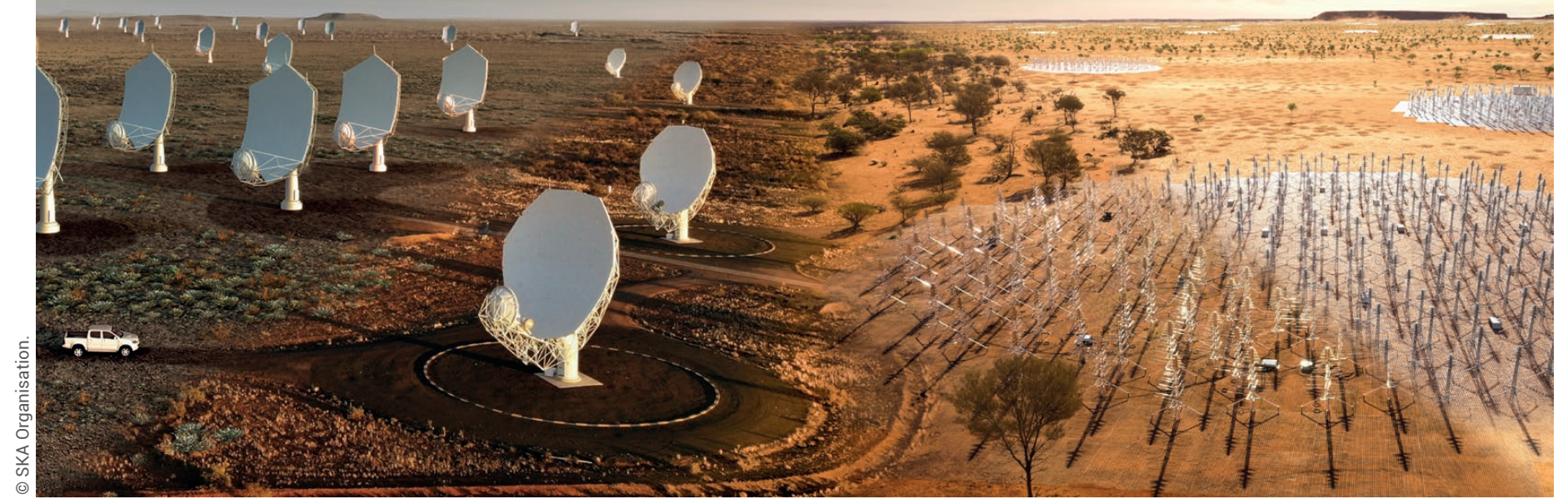

2. Impression d'artiste des paraboles de SKA-MID en Afrique du Sud (à gauche) et des antennes de SKA-LOW en Autralie (à droite).

fabrication et de fonctionnement (liés en particulier à la consommation d'énergie et à la capacité de calcul) qui sont aujourd'hui prohibitifs pour la communauté scientifique. C'est pour cette raison que SKA1-MID sera constitué par un réseau de paraboles qui, à partir des années 1960, ont été le type d'antennes le plus utilisé en radioastronomie à haute et moyenne fréquence.

Des radiotélescopes qui permettent de se préparer à SKA sont opérationnels aujourd'hui, aussi bien sur ses sites futurs (appelés dans ce cas "précurseurs ") qu'ailleurs (définis alors comme "éclaireurs"). En France, une station de LOFAR (éclaireur européen de SKA1-LOW) et l'éclaireur français NenuFAR (opérant entre 10 et $85 \mathrm{MHz}$ ) sont installés à la Station de Radioastronomie de Nançay.

\section{Des observables uniques pour explorer le cosmos}

Parmi tous les sujets qui pourront être abordés par SKA, et dont une vision d'ensemble est disponible au travers de l'ouvrage Advancing Astrophysics with the Square Kilometre Array ${ }^{(\mathrm{d})}$, nous présentons dans la suite quelques applications pour lesquelles cet observatoire sera un instrument clé.

\section{Les gaz atomiques \\ d'hydrogène neutre}

Depuis les années 1950, les astronomes ont pu commencer à observer le gaz diffus composé d'hydrogène atomique neutre. Comme prévu théoriquement en 1945 par l'astronome néerlandais Van de Hulst, la transition atomique entre les deux sousniveaux de la structure hyperfine du niveau fondamental de l'atome d'hydrogène est en effet responsable de l'émission de photons à une fréquence (longueur d'onde) de $1420 \mathrm{MHz}(21 \mathrm{~cm})$. SKA, qui pourra mener différents types d'études sur l'émission de la raie d'HI dans les galaxies (e.g. [2], [3]), est attendu dans le domaine cosmologique, surtout pour sa capacité à étudier la distribution du gaz d'hydrogène atomique au cours de l'époque de formation des premiers objets lumineux de l'Univers, dont nous allons résumer les principales étapes.

Environ 380000 ans après le Big Bang, l'expansion cosmique avait suffisamment refroidi l'Univers pour que les électrons et les protons primordiaux forment des atomes neutres (la " recombinaison ") et que la lumière $\mathrm{du}$ fond diffus cosmologique puisse se propager et être détectée par des instruments submillimétriques (e.g. le satellite Planck de l'ESA [4]). L'Univers était alors plongé dans l'obscurité, car aucune source lumineuse ne s'était encore formée. Au cours de ces "âges sombres ", la gravité amplifia les petites fluctuations de densité primordiale, essentiellement de la matière noire, qui ont été observées dans le fond diffus cosmologique, et provoqua l'effondrement des nuages de gaz d'hydrogène neutre dans ces halos noirs. Quand l'Univers était âgé d'un peu moins de 200 millions d'années, les régions les plus denses formèrent finalement les premières étoiles et galaxies. Leur lumière ultraviolette ionisa le gaz neutre restant et, environ un milliard d'années après le Big Bang, suffisamment de sources lumineuses s'étaient formées pour que l'hydrogène intergalactique soit totalement ionisé, en marquant la fin de cette époque dite de la "réionisation ».

En raison de l'expansion cosmique et donc de "l'étirement " de la longueur d'onde de la lumière durant son trajet vers nous, les photons émis à $21 \mathrm{~cm}$ pendant cette phase de vie de l'Univers possèdent aujourd'hui une longueur d'onde d'autant plus grande qu'ils ont été émis tôt, variant entre environ 4 et 1,5 m (entre le début et la fin de l'époque de la réionisation). Des sources radio d'avant-plan contamineront ces signaux cosmiques, mais elles pourront être filtrées grâce au fait que, contrairement au signal de la réionisation, leur flux lumineux ne change pas rapidement en fonction de la longueur d'onde.

Au travers de sa sensibilité et de sa couverture spectrale, SKA sera le premier instrument capable de cartographier la distribution du gaz d'hydrogène tout au long de l'époque de la réionisation et dans les phases finales d'effondrement des nuages de gaz avant la naissance des astres, en nous donnant des contraintes uniques sur l'histoire globale de la formation des structures [5].

\section{Le magnétisme cosmique}

Le rayonnement synchrotron, engendré par des électrons relativistes décrivant un mouvement de spirale autour des lignes de champ magnétique, est d'importance particulière dans de nombreux domaines de l'astronomie. En bande radio, il est par 
exemple responsable de la plus grande part de l'émission radio des noyaux galactiques actifs et, au-dessous de $30 \mathrm{GHz}$, il domine l'émission radio des galaxies qui forment des étoiles avec un taux similaire à celui de notre Voie lactée. En plus de nous permettre d'identifier la présence d'électrons de très haute énergie et d'étudier l'origine physique de leur accélération (phénomènes explosifs, supernovae, éjections de matière par des trous noirs actifs, chocs...), l'observation $\mathrm{du}$ rayonnement synchrotron en bande radio est un outil très puissant pour étudier le magnétisme cosmique, grâce en particulier à sa polarisation linéaire. Nous savons en effet que, quand ce rayonnement traverse un milieu magnétisé, sa direction de polarisation subit une rotation à cause de l'effet Faraday. L'angle de rotation est proportionnel à l'intensité du champ magnétique traversé ou, pour être plus précis, à sa composante le long de la direction de propagation de la lumière. Puisque des observations radio à plusieurs fréquences nous permettent de mesurer l'angle de rotation du signal polarisé, nous pouvons en déduire des informations sur le champ magnétique qui remplit l'espace traversé par la radiation. SKA augmentera d'au moins un facteur 300 le nombre de sources synchrotron connues au travers desquelles effectuer des mesures de rotation. Nous pourrons alors cartographier de plus en plus finement la distribution des champs magnétiques dans l'Univers.

La présence de champs magnétiques caractérise en effet tous les objets du cosmos : les planètes, les étoiles, les nébuleuses gazeuses des milieux interstellaire et intergalactique, jusqu'aux amas de galaxies. Grâce à une découverte récente et en accord avec les prévisions des simulations numériques [6], nous savons aujourd'hui que même les filaments très raréfiés de la " toile cosmique " (terme qui décrit la topologie de la distribution à grande échelle de la matière dans l'Univers, voir la figure 3) sont faiblement magnétisés, avec une intensité de quelques centaines de milliardième du champ magnétique terrestre. Par ailleurs, les objets célestes peuvent aussi atteindre des niveaux d'aimantation extrêmement élevés, comme dans le cas des pulsars, des étoiles à neutrons qui feront l'objet d'études approfondies avec SKA et qui sont caractérisées par des champs magnétiques des millions de millions de fois plus intenses que celui de la Terre [7].

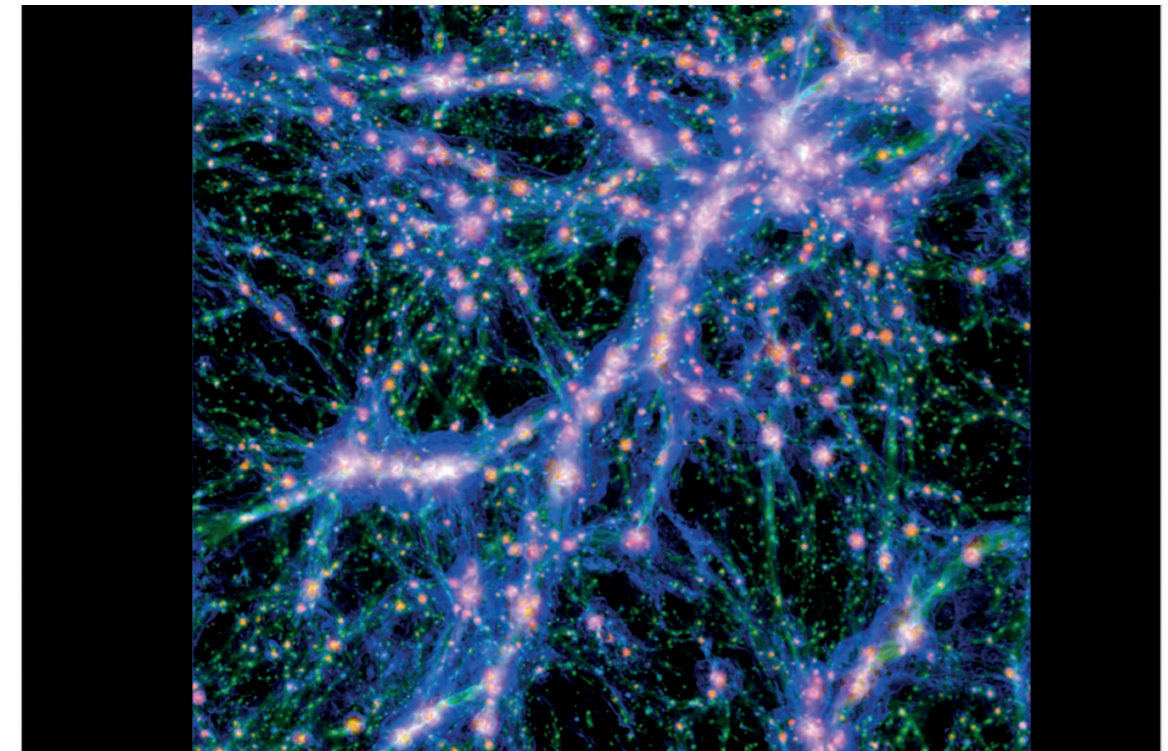

3. Simulation numérique dans une boite cubique de (100 Mparsec) ${ }^{3}$, reproduisant les émissions $X$ et radio attendues de la structure à grande échelle de l'Univers. La couleur orange correspond au rayonnement de freinage thermique émis en bande $X$ par le gaz chaud et diffus dans les filaments de la toile cosmique et, surtout, dans les amas de galaxies localisés aux croisements de ces filaments. La couleur bleue est associée au rayonnement synchrotron en bande radio engendré par les électrons relativistes dans les champs magnétiques, dont la distribution est représentée en vert.

Adapté de F. Vazza et al., "Detecting shocked intergalactic gas with X-ray and radio observations", A\&A 627 (2019) 5.

Les recherches visant à comprendre l'origine et l'évolution du magnétisme cosmique sont de très grande actualité. Par exemple, les astronomes s'interrogent sur le rôle indéniable que ces champs doivent jouer dans l'évolution de la matière interstellaire, en sachant qu'il sera impossible de comprendre finement la formation des étoiles et des planètes sans en tenir compte.

\section{Vers l'inconnu :}

l'exemple des sursauts radio rapides

Tous les astronomes explorent l'Univers en espérant tomber un jour sur de " l'inconnu ». C'est ce qui est arrivé en 2007 à D. Lorimer qui, en réexaminant des données de 2001 du radiotélescope de Parkes (antenne de $64 \mathrm{~m}$ en Australie), découvrit un nouveau type de source radio transitoire : un sursaut d'émission radio très intense et de durée inférieure à quelques millièmes de seconde. Ce type de source, qui peut émettre en ces intervalles de temps très courts plus d'énergie que le Soleil en 80 ans, fut baptisé «sursaut radio rapide " ou "fast radio burst" (FRB) en anglais. Depuis, plus de cent nouveaux FRB ont été annoncés(e), parmi lesquels plus de la moitié l'ont été au cours des deux dernières années grâce à deux télescopes construits pour préparer la voie à SKA. Le premier est ASKAP, en Australie, opérant entre 700 et $1800 \mathrm{MHz}$ et capable non seulement de détecter efficacement les $F R B$, mais aussi d'en dériver la position dans le ciel avec une précision très élevée. Le second est CHIME, au Canada, premier instrument basse fréquence (400-800 MHz), qui a capté des FRB alors qu'il n'était encore qu'en phase de mise en service.

Il reste encore à éclaircir l'existence de deux différentes classes de sursauts radio rapides : ceux récurrents et ceux qui ne se répètent jamais. Le fait de ne pas réobserver certaines sources pourrait être lié à des variations de la luminosité radio émise au cours du temps. Mais il est aussi tout à fait possible que différentes classes de FRB existent, selon plusieurs phénomènes physiques plus ou moins exotiques qui pourraient être associés à l'interaction, à la coalescence ou à l'effondrement d'objets compacts, aux restes de supernovae, aux noyaux galactiques actifs, ou encore aux hypothétiques cordes cosmiques.

Les observations indiquent que tous les $F R B$ connus $^{(\mathrm{f})}$ (sauf un, comme on le verra) sont d'origine extragalactique. Un délai est observé entre les temps d'arrivée des différentes composantes en fréquence du sursaut, les photons moins énergétiques arrivant après ceux émis aux fréquences plus élevées. Ce délai, appelé «mesure de 
$>>$

dispersion ", produit lorsque les ondes électromagnétiques traversent le plasma cosmique, est un indicateur direct du nombre d'électrons libres par unité de surface entre l'observateur et la source qui a émis le FRB. Les dispersions importantes mesurées pour les sursauts observés indiquent qu'ils ont traversé trop de matière pour se trouver à l'intérieur de notre Galaxie. La localisation, à ce jour, de six FRB dans des galaxies à des distances de plusieurs milliards d'annéeslumière confirme l'hypothèse extragalactique. Parmi ces six objets, l'un est un FRB récurrent qui se trouve dans une galaxie naine avec un taux de formation stellaire important; en revanche, il n'y a pas eu de répétitions observées des autres FRB qui se trouvent dans des galaxies plus massives, mais avec des taux de formation stellaire significativement plus faibles.

Les propriétés de ces galaxies suggèrent que les sources hypothétiques des sursauts radio rapides sont des "magnétars ", étoiles à neutrons disposant d'un champ magnétique extrêmement intense. L'origine physique des magnétars est à son tour à l'étude, et plusieurs théories ont été proposées. En mettant ensemble tous les morceaux du puzzle, on pense aujourd'hui que les magnétars associés aux FRB qui se répètent ou pas pourraient résulter d'explosions de supernovae avec des énergétiques différentes, supralumineuses dans un cas, standard dans l'autre. En accord avec l'hypothétique association entre $F R B$ et magnétars, une équipe de chercheurs a annoncé en mai 2020 l'observation d'un premier candidat de $F R B$ galactique associé à un sursaut d'émission en rayons $\mathrm{X}$ en provenance d'une source connue de rayons gamma. L'hypothèse la plus probable qui expliquerait ces propriétés multi-longueurs d'onde est que ce candidat FRB soit produit par un magnétar dans notre Galaxie.

Il est important cependant de souligner que d'autres hypothèses sur les sources d'émission des FRB restent tout à fait ouvertes, en particulier l'effondrement et la coalescence d'objets compacts dans le cas des sources non récurrentes, ainsi que des systèmes binaires dans le cas des FRB qui se répètent. SKA prévoit d'observer des milliers de sursauts radio rapides.

Ainsi, les astronomes pourront non seulement identifier la (ou les) mystérieuse(s) origine(s) physique(s) des FRB, mais aussi, au travers de la mesure de dispersion, utiliser ces derniers pour cartographier la distribution du gaz dans les halos des galaxies [8] ou dans l'espace intergalactique. À ce propos, l'équipe de "FRB hunters" d'ASKAP dirigée par J.-P. Macquart a publié en mai 2020 un résultat très marquant, en obtenant pour la première fois une mesure de la densité de matière ordinaire dans l'Univers, qui est en accord parfait avec les prévisions cosmologiques.

\section{Références}

1 P. Zarka et al., Radioastronomie basses fréquences, École thématique du CNRS, Goutelas, Loire (2007).

2• F. Combes, La matière noire dans I'Univers, Collège de France / Fayard (2015).

F. Combes, « La matière noire, une sombre affaire », Reflets de la physique 51 (2016) 4-10.

3. C. Pichon et T. Sousbie, « Cartographier l'Univers », Dossier Pour la Science, 71 (avril-juin 2011).

4 - N. Aghanim et H. Dole, "Les résultats cosmologiques de la mission Planck », Reflets de la physique 64 (2020) 4-10.

5• A. Loeb, «L'Univers à l'âge des ténèbres », Dossier Pour la Science, 71 (avril-juin 2011).

6• Communiqué de presse de l'Observatoire de la Côte d'Azur :

https://cutt.ly/oca-eu-aurorecosmique-eclaire-la-connexion-entre-deux-amas-de-galaxies

7• I. Cognard, «Les pulsars radio : 50 ans de découvertes ! », Reflets de la Physique, 59 (2018) 26-31.

8. Communiqué de presse de l'ESO : www.eso.org/public/france/news/eso1915/?lang

(a) Liste des pays et instituts participant à sa construction : www.skatelescope.org

(b) Cf. le tableau 1 dans P. Dewdney et al., "SKA1 System BaselineV2”, SKA-TEL-SKO-0000002 (2016). À noter que le grand radiotélescope international ALMA n'est pas inclus dans ce tableau, car il est complémentaire à SKA en termes de couverture du spectre électromagnétique. ALMA capte en effet des ondes de fréquences plus élevées (35-950 GHz).

(c) Un interféromètre étant constitué par un nombre d'antennes limité et une surface de récolte de la lumière non continue, après avoir corrélé les signaux captés par chaque couple d'antennes, nous ne disposons que d'échantillons de la transformée de Fourier de l'intensité observée du ciel. Un ensemble d'opérations mathématiques doit ainsi être effectué afin d'obtenir une image du ciel.

(d) Une version électronique des deux volumes est disponible à l'adresse : https://cutt.ly/SKA .

(e) Une liste des FRB découverts et de leurs propriétés est disponible à l'adresse : www.frbcat.org

(f) Une liste est mise à jour régulièrement à l'adresse : https://frbtheorycat.org/index.php/Main_Page 